\title{
The C-terminus of the B-chain of human insulin-like peptide 5 is critical for cognate RXFP4 receptor activity
}

\author{
Nitin A. Patil ${ }^{1,2}$, Ross A. D. Bathgate ${ }^{1,3}$, Martina Kocan ${ }^{4}$, Sheng Yu Ang, ${ }^{4}$ Julien Tailhades ${ }^{1}$, Frances \\ Separovic $^{2}$, Roger Summers ${ }^{4}$, Johannes Grosse ${ }^{5}$, Richard A. Hughes ${ }^{6}$, John D. Wade ${ }^{1,2}$, Mohammed Akhter \\ Hossain $^{1,2 *}$ \\ ${ }^{1}$ Florey Institute for Neuroscience \& Mental Health, ${ }^{2}$ School of Chemistry, Bio21 Institute, ${ }^{3}$ Department of \\ Biochemistry and Molecular Biology, ${ }^{6}$ Department of Pharmacology, University of Melbourne; ${ }^{4}$ Monash \\ Institute of Pharmaceutical Sciences, Parkville, Victoria, Australia; ${ }^{5}$ Takeda Cambridge Ltd., Cambridge, \\ United Kingdom
}

\section{Address correspondence to}

Dr. Mohammad Akhter Hossain

Email: akhter.hossain@unimelb.edu.au

Tel: +61383440414

Professor John Wade

john.wade@florey.edu.au

Howard Florey Research Laboratories

Florey Institute for Neuroscience \& Mental Health

University of Melbourne, Melbourne

Victoria, Australia

\section{Conflict of Interest}

The authors declare that they have no conflict of interest.

\section{Research involving Human Participants and/or Animals}

"This article does not contain any studies with human participants performed by any of the authors."

"This article does not contain any studies with animals performed by any of the authors."

"This article does not contain any studies with human participants or animals performed by any of the authors." 


\begin{abstract}
Insulin-like peptide 5 (INSL5) is an orexigenic peptide hormone belonging to the relaxin family of peptides. It is expressed primarily in the L-cells of the colon and has a postulated key role in regulating food intake. Its $\mathrm{G}$ protein-coupled receptor, RXFP4, is a potential drug target for treating obesity and anorexia. We studied the effect of modification of the C-terminus of the A and B-chains of human INSL5 on RXFP4 binding and activation. Three variants of human INSL5 were prepared using solid phase peptide synthesis and subsequent sequential regioselective disulfide bond formation. The peptides were synthesized as C-terminal acids (both Aand B-chains with free C-termini, i.e. the native form), amides (both chains as the C-terminal amide) and one analogue with the C-terminus of its A-chain as the amide and the C-terminus of the B-chain as the acid. The results showed that $\mathrm{C}$-terminus of the $\mathrm{B}$-chain is more important than that of the A-chain for RXFP4 binding and activity. Amidation of the A-chain C-terminus does not have any effect on the INSL5 activity. The difference in RXFP4 binding and activation between the three peptides is believed to be due to electrostatic interaction of the free carboxylate of INSL5 with a positively charged residue (s), either situated within the INSL5 molecule itself or in the receptor extracellular loops.
\end{abstract}

Key words: insulin-like peptide 5, INSL5, relaxin, peptide 


\section{Introduction}

Insulin-like peptide 5 (INSL5) is a peptide hormone member of the insulin superfamily that comprises insulin, insulin-like growth factors I and II (IGFI-II), relaxins 1-3 and insulin like peptides 3-6 (Bathgate et al. 2013). Similar to other family peptides, INSL5 is expressed as a single-chain prohormone that is converted to the mature peptide by formation of three disulfide bonds (one intra-chain and two inter-chains) with two chains (Achain and B-chain) (Hossain et al. 2008). Its tertiary structure has been determined using solution NMR spectroscopy which shows a high degree of similarity to known structures from other members of the family, particularly within the regions held together by the three disulfide bonds (Haugaard-jönsson et al. 2009). INSL5 binds and activates its cognate receptor known as relaxin-family peptide receptor-4 (RXFP4) (Conklin et al. 1999; Liu et al. 2005). The mRNA of INSL5 is primarily expressed in the colonic L-cells with minor expression in other organs (Grosse et al. 2014). INSL5 has recently been discovered to play an important role in appetite regulation (Grosse et al. 2014). These findings suggest that RXFP4 agonists and antagonists may be prospective therapeutic agents for appetite-related disorders such as cachexia, anorexia and obesity. Considering these important applications, a critical goal is to understand the key aspects of ligand-receptor (INSL5-RXFP4) interaction.

A preliminary structure-activity relationship (SAR) study of mouse INSL5 showed that the carboxyl group at the C-terminus (free C-terminus) of INSL5 plays an important role in the interaction with its receptor (Belgi et al. 2011). Similarly, the free C-terminal (- $\mathrm{COOH}$ group) of the B-chain of the related peptide relaxin-3 plays an important role in interacting with its receptor RXFP3, whereas the A-chain C-terminus can be amidated (Shabanpoor et al. 2013a). Since the receptors for both relaxin-3 and INSL5 are similar, we hypothesized that the C-terminus of the INSL5 B-chain, and not that of the INSL5 A-chain, may be crucial for RXFP4 activity. In order to investigate the influence of C-terminus of both chains of human INSL5 (hINSL5), three analogues (Fig. 1) were prepared using solid phase peptide synthesis followed by sequential regioselective folding, and further assayed for RXFP4 binding, cAMP activity and ERK1/2 activity.

\section{Materials and Methods}


Materials: All 9-fluoroenylmethoxycarbonyl (Fmoc) protected L- $\alpha$-amino acids and O-(6-chlorobenzotriazol-1yl)-N,N,N',N'-tetramethyluronium hexafluorophosphate (HCTU) were obtained from GL Biochem (Shanghai, China). Fmoc-Cys(Acm)-Ac-S-TentaGel $(0.23 \mathrm{mmol} / \mathrm{g})$ resin was obtained from Rapp Polymere GmbH (Tübingen, Germany). Fmoc-Trp(Boc)-PHB-TentaGel (0.23 mmol/g) and Fmoc-PAL-PEG-PS (0.19 mmol/g) resins were purchased from Applied Biosystems (Melbourne, Australia). All peptide synthesis solvents were obtained from Merck (Melbourne, Australia). 2, 2'-dipyridyl disulfide (DPDS) was from Fluka (Bucha, Switzerland) and Trifluoromethanesulfonic acid (TFMSA) from MP Biomedicals (Sydney, Australia). All other reagents for peptide synthesis and bovine serum albumin (BSA) and 4-(2-hydroxyethyl)-1-piperazine ethanesulfonic acid (HEPES) were obtained from Sigma-Aldrich (Sydney, Australia).

Peptide synthesis: Linear peptides with a C-terminal amide were assembled on Fmoc-PAL-PEG-PS resin and the A- and B-chains with C-terminal acid were synthesised using Fmoc-Cys)-Ac-S-TentaGel and Fmoc$\operatorname{Trp}(\mathrm{Boc})-\mathrm{PHB}-\mathrm{TentaGel}$, respectively. All the B-chain peptides were synthesised using a CEM Liberty ${ }^{\mathrm{TM}}$ microwave peptide synthesizer (AI Scientific, Scarborough, Australia), whereas all the A-chain peptides were synthesized by manual stepwise solid phase synthesis. Each peptide was synthesised on a 0.1-mmol scale with excess of Fmoc-protected amino acids, HCTU and DIPEA (5:5:10). Fmoc removal was carried out using 20\% piperidine in DMF. The peptides were cleaved from solid support using a cocktail of TFA:anisole:DODT:TIPS (94:3:2:1, v/v) for $2 \mathrm{hr}$. Combination of the two chains was carried out using sequential regioselective disulfide bond formation as previously described (Shabanpoor et al. 2013b). The crude peptides were analyzed and purified by RP-HPLC on Phenomenex Gemini columns $(4.6 \times 250 \mathrm{~mm}, \mathrm{C} 18,5 \mu \mathrm{m} ; 19 \times 150 \mathrm{~mm}, \mathrm{C} 18,5 \mu \mathrm{m})$ using $\mathrm{H}_{2} \mathrm{O}$ and a gradient of acetonitrile with $0.1 \%$ TFA as solvent $\mathrm{A}$ and $\mathrm{B}$, respectively. The characterization of peptides was carried using MALDI-TOF/TOF mass spectroscopy (Bruker Daltonics, Germany) with sinapinic acid as matrix.

Circular dichroism (CD) spectroscopy: The secondary structural changes of the peptides were measured by recording their CD spectra on JASCO model J815 spectropolarimeter as previously described (Shabanpoor et al. 2013a; Najbar et al. 1997). The CD spectra were recorded in $10 \mathrm{mM}$ phosphate buffered saline (PBS) with peptide concentrations made up to $0.2 \mu \mathrm{g} / \mu \mathrm{l}$.

Binding assays: The ability of the peptides to bind to the RXFP4 receptor was measured using Chinese hamster ovary CHO-K1 cells stably expressing human RXFP4 (CHO-K1-RXFP4) (Belgi et al. 2013). The transfected cells were plated out at 50,000 cells per well per $200 \mu \mathrm{L}$ in a 96 -well ViewPlate. Competition binding 
experiments were performed with $5 \mathrm{nM}$ of europium-labeled $\mathrm{Eu}(\mathrm{A})$-mouse INSL5 acid in the presence of increasing amounts of peptides following the protocol described previously (Belgi et al. 2013). Fluorescence was measured at excitation wavelength of $340 \mathrm{~nm}$ and emission wavelength of $614 \mathrm{~nm}$ on a BMG POLARstar plate reader (BMG Labtech). Each concentration point was measured in triplicate, in three independently performed experiments. GraphPad PRISM 6 (GraphPad Inc., San Diego, CA) was used to analyze the receptorbinding assays data that are expressed as mean \pm SEM.

cAMP activity assays: The receptor activation by the peptides was assessed based on their ability to inhibit cAMP activity in CHO-K1-RXFP4 transfected with a pCRE (cAMP Response Element) $\beta$-galactosidase reporter plasmid (Scott et al. 2006; Belgi et al. 2011). CHO-K1-RXFP4 cells were plated out at 8,000 per well per $100 \mu \mathrm{L}$ in a Corning cell bind 96-well plate on day 1 and transfected with the pCRE $\beta$-galactosidase reporter plasmid on day 2. One day later the cells were stimulated with $1 \mu \mathrm{M}$ of forskolin plus or minus increasing concentrations of each peptide as previously described (Belgi et al. 2013). cAMP activity is assessed with a $\beta$ galactosidase colorimetric assay measuring absorbance at $570 \mathrm{~nm}$ on a Benchmark Plus microplate spectrophotometer (Bio-Rad Laboratories, Sydney, Australia). Each concentration point was measured in triplicate, and each experiment was performed independently at least three times. GraphPad PRISM 6 (GraphPad Inc., San Diego, CA) was used to analyze the cAMP inhibition assays data that are expressed as mean \pm SEM.

ERK 1/2 phosphorylation AlphaScreen SureFire assay: CHO-K1-RXFP4 cells were plated out at 50,000 cells per well per $100 \mu \mathrm{L}$ in a 96-well plate and grown overnight in complete media (DMEM/F12) at $37^{\circ} \mathrm{C}$ in a humidified atmosphere of $5 \% \mathrm{CO}_{2}$. Cells were washed twice with PBS and then serum-starved for $6 \mathrm{~h}$ followed by stimulation with an increasing concentration of each peptide, serum free DMEM/ F12 as vehicle control, or $10 \% \mathrm{FBS}$ in DMEM/F12 as positive control for $5 \mathrm{~min}$ at $37^{\circ} \mathrm{C}$. Following stimulation, the cells were lysed using $100 \mu \mathrm{L}$ of lysis buffer and frozen at $-20^{\circ} \mathrm{C}$ according to the assay manufacturer's instructions. For detection of ERK1/2 kinase phosphorylation, the thawed cell lysate, $4 \mu \mathrm{L}$, was transferred to white 384-well microplates (Proxiplates), and $5 \mu \mathrm{L}$ of the combination buffer with AlphaScreen donor beads (40 parts reaction buffer, 10 parts activation buffer, and 1 part acceptor beads) was added. The plate was incubated for $2 \mathrm{~h}$ at $23^{\circ} \mathrm{C}$ in the dark on an oscillating platform. Subsequently, $2 \mu \mathrm{L}$ of the dilution buffer with AlphaScreen acceptor beads (20 parts dilution buffer and 1 part donor beads) was added. The plate was incubated for $2 \mathrm{~h}$ at $23^{\circ} \mathrm{C}$ in the dark on the oscillating platform one more time. The AlphaScreen signal (counts per second) was measured in 384-well 
microplates (Proxiplates) on an EnVision Multilabel Plate Reader (PerkinElmer Life and Analytical Sciences) with excitation at $680 \mathrm{~nm}$ and emission at 520 to $620 \mathrm{~nm}$. Results are the mean \pm SEM of three independent experiments.

\section{Result and discussion}

To determine the effect of the C-termini of hINSL5 on the receptor binding and activity, three hINSL5 analogues, hINSL5-acid (both A-and B-chains C-termini acid), hINSL5-amide (both A-and B-chains C-termini amide), and hINSL5-B-acid (A-chain with C-terminus amide and B-chain with C-terminus acid) were synthesized using regioselective disulfide bond formation protocol (Hossain et al. 2008). We have previously shown that INSL5 is the most difficult peptide to synthesize among the members of the insulin-relaxin superfamily (Hossain et al. 2008). Considering the challenges in the solid phase peptide synthesis of the Achain, we carried out the synthesis manually with each coupling performed with 4 equivalent excess of Fmocamino acids, HCTU and DIEA. The use of pseudoproline Leu-Ser( $\left.\psi^{\mathrm{MeMe}} \mathrm{Pro}\right)-\mathrm{OH}$ enabled the successful synthesis of the A-chain (Hossain et al. 2008). The post-synthetic sequential disulfide bond formation was employed as reported in our previous work (Hossain et al. 2006). The successful synthesis of all three analogues was confirmed by MALDI-TOF mass spectrometric and amino acid analysis (Table 1). The overall yields of the hINSL5 analogues in this study (based on the purified B-chain) shown in Table 1, again confirmed that amide peptides (C-termini amides) were easier to assemble compared with acid peptides (C-termini acid).

The binding and cAMP assays showed that hINSL5-amide (both chain amide) has markedly lower affinity for RXFP4 compared with hINSL5-acid (both chain acid) and importantly showed minimal cAMP activity at concentrations up to $1 \mu \mathrm{M}$ (Fig 2, Table 2). These results are consistent with our previous study with the murine homologue (Belgi et al. 2011). Activation of RXFP4 also induces ERK1/2 which is another signalling molecule involved in RXFP4-mediated cellular pathways (Belgi et al. 2013). Thus, we also tested all the analogues on human RXFP4 receptor in the ERK1/2 phosphorylation assay (Fig 3). Consistent with cAMP results, hINSL5acid showed clear dose dependent activation of ERK1/2 phosphorylation whereas the hINSL5-amide demonstrated no activation at concentrations up to $1 \mu \mathrm{M}$ (Fig. 3). Finally, to determine the particular effect of the C-termini of individual chains (A-chain or B-chain) we tested binding and activity of hINSL5-B-acid (B acid/A amide). Interestingly, hINSL5-B-acid was found to be almost equipotent to hINSL5-acid both in cAMP 
(Fig. 2; Table 2) and pERK assays (Fig. 3) and also demonstrated equivalent binding affinity for RXFP4 (Fig 2 , Table 2). This result indicates that a free B-chain C-terminus is important for high RXFP4 binding and activity. A similar trend was observed with human relaxin-3 and its RXFP3 interaction (Shabanpoor et al. 2013a) which reflects the fact that both RXFP3 and 4 have considerable structural similarities. On the other hand, we have recently shown that modifications to the C-terminus of either of the A- or B-chain of human relaxin-2 have no effect on RXFP1 activity (Haugaard-Kedström et al. 2015). However, the RXFP1 receptor has a complex structure with a large ectodomain and the mode of ligand interaction is completely different to RXFP3 and 4 (Hossain et al. 2008; Kong et al. 2010).

We carried out CD spectroscopy analysis of the peptides in order to obtain an insight into their secondary structures. Two of the analogues (INSL5 amide and INSL5-B-acid) were found to aggregrate in solution (both in phosphate and TFE buffers). Therefore, it was not possible to compare their secondary structures. However, based on our previous studies with related truncated INSL5 or relaxin-3 analogues (Belgi et al. 2011; Shabanpoor et al. 2013a), we do not expect that a small change at the C-termini $\left(-\mathrm{COOH}\right.$ to $\left.-\mathrm{NH}_{2}\right)$ would cause dramatic change in overall structural fold. The combined loss of binding and activation in hINSL5 amide suggests that the C-terminal Trp is involved in receptor binding similar to what we have previously demonstrated for mouse INSL5 (Belgi et al. 2011). Furthermore, these data suggest there may be an electrostatic interaction of the free carboxylate of the C-terminal Trp with positively charged residue (s) in the RXFP4 receptor. Indeed, a recent study has demonstrated that $\operatorname{Arg}^{\mathrm{B} 23}$ of the INSL5 B-chain interacts via an electrostatic interaction with both Glu ${ }^{100}$ and Asp ${ }^{104}$ of RXFP4 (Wang et al. 2014); the role of Trp ${ }^{\text {B24 }}$ was not investigated.

\section{Conclusion}

We have chemically prepared three complex two-chain, three-disulfide-bond hINSL5 peptides possessing either or both C-terminal acids or amides. We observed that the free carboxyl group at the C-terminus of the B chain of hINSL5 is crucial for high RXFP4 affinity and activity. The C-terminal amide peptides are easier to synthesize compared with the acid peptides. Considering the difficulty in synthesis and poor recovery of native INSL5 (INSL5-acid), INSL5-B-acid will be an important template for future structure-function studies. 


\title{
Acknowledgement
}

This research was partly funded by NHMRC (Australia) project grants $(1023321,1065481,1023078)$ to M. A. H, R. A. D. B and J. D. W and ARC linkage grant (LP120100654) to R.A.H. We are grateful to Tania Ferraro and Sharon Layfield for assistance with biochemical assays. During these studies, M.A.H. was the recipient of Florey Foundation Fellowships. R.A.D.B. is an NHMRC Senior Research Fellow, and J.D.W. is an NHMRC Principal Research Fellow. Studies at the FINMH were supported by the Victorian Government's Operational Infrastructure Support Program.

\begin{abstract}
Abbreviations used
INSL5, insulin-like peptide 5; mINSL5, mouse insulin-like peptide 5; hINSL5, human insulin-like peptide 5; IGFI, insulin-like growth factor I; IGFII, insulin-like growth factor II; INSL3, insulin-like peptide 3; INSL4, insulin-like peptide 4; INSL6, insulin-like peptide 6; RXFP3, relaxin-family peptide receptor 3; RXFP4, relaxin-family peptide receptor 4; Boc, tert-butoxycarbonyl; Acm, acetamidomethyl; cAMP, 3',5'-cyclic adenosine monophosphate; PEG-PS, poly(ethylene glycol)-polystyrene; CD, circular dichroism; CHO, Chinese hamster ovary; DMF, dimethylformamide; DIEA, diisopropylethylamine; Fmoc, 9-fluoroenylmethoxycarbonyl; GPCR, G-protein coupled receptor; HCTU, O-(6-chlorobenzotriazol-1-yl)-N,N,N',N'-tetramethyl uronium hexafluorophosphate; INSLs, insulin-like peptides; RXFP, relaxin-family peptide; tBu, tert-butyl; TIPS, triisopropylsilane; TFA, trifluoroacetic acid; TFMSA, trifluoromethanesulfonic acid; DODT, 3,6-dioxa-1,8octanedithiol; DPDS, 2,2'-dipyridyldisulfide; BSA, bovine serum albumin; HEPES, 4-(2-hydroxyethyl)-1piperazineethanesulfonic acid; ERK, extracellular-signal-regulated kinase; DMEM, Dulbecco's modified Eagle's medium.
\end{abstract}




\section{References}

Bathgate RAD, Halls ML, van der Westhuizen ET, Callander GE, Kocan M, Summers RJ (2013) Relaxin family peptides and their receptors. Physiology Reviews 93:405-480.

Belgi A, Bathgate RA, Kocan M, Patil N, Zhang S, Tregear GW, Wade JD, Hossain MA (2013) Minimum active structure of insulin-like peptide 5. J Med Chem 56 (23):9509-9516.

Belgi A, Hossain MA, Shabanpoor F, Chan L, Zhang S, Bathgate RAD, Tregear GW, Wade JD (2011) Structure and Function Relationship of Murine Insulin-like Peptide 5 (INSL5): Free C-Terminus is essential for RXFP4 receptor binding and activation. Biochemistry 50 (39):8352-8361.

Conklin D, Lofton-Day CE, Haldeman BA, Ching A, Whitmore TE, Lok S, Jaspers S (1999) Identification of INSL5, a new member of the insulin superfamily. Genomics 60 (1):50-56.

Grosse J, Heffron H, Burling K, Akhter Hossain M, Habib AM, Rogers GJ, Richards P, Larder R, Rimmington D, Adriaenssens AA, Parton L, Powell J, Binda M, Colledge WH, Doran J, Toyoda Y, Wade JD, Aparicio S, Carlton MBL, Coll AP, Reimann F, O’Rahilly S, Gribble FM (2014) Insulin-like peptide 5 is an orexigenic gastrointestinal hormone. Proceedings of the National Academy of Sciences 111 (30):11133-11138.

Haugaard-jönsson LM, Hossain MA, Daly NL, Craik DJ, Wade JD, Rosengren KJ (2009) Structure of human insulin-like peptide 5 and characterization of conserved hydrogen bonds and electrostatic interactions within the relaxin framework. The Biochemical journal 419 (3):619-627.

Haugaard-Kedström LM, Hossain MA, Daly NL, Bathgate RAD, Rinderknecht E, Wade JD, Craik DJ, Rosengren KJ (2015) Solution structure, aggregation behavior, and flexibility of human relaxin-2. ACS chemical biology 10 (3):891-900.

Hossain AM, Bathgate RAD, Kong CK, Shabanpoor F, Zhang S, Haugaard-Jönsson LM, Rosengren KJ, Tregear GW, Wade JD (2008) Synthesis, conformation, and activity of human insulin-like peptide 5 (INSL5). ChemBioChem 9 (11):1816-1822.

Hossain MA, Lin F, Zhang S, Ferraro T, Bathgate RA, Tregear GW, Wade JD (2006) Regioselective Disulfide Solid phase synthesis, chemical characterization and in vitro receptor binding activity of equine relaxin. International Journal of Peptide Research and Therapeutics 12 (3):211-215.

Kong RC, Shilling PJ, Lobb DK, Gooley PR, Bathgate RA (2010) Membrane receptors: structure and function of the relaxin family peptide receptors. Molecular and cellular endocrinology 320 (1-2):1-15.

Liu C, Kuei C, Sutton S, Chen J, Bonaventure P, Wu J, Nepomuceno D, Kamme F, Tran DT, Zhu J, Wilkinson T, Bathgate R, Eriste E, Sillard R, Lovenberg TW (2005) INSL5 is a high affinity specific agonist for GPCR142 (GPR100). The Journal of biological chemistry 280 (1):292-300.

Najbar LV, Craik DJ, Wade JD, Salvatore D, McLeish MJ (1997) Conformational Analysis of LYS(11-36), a Peptide Derived from the $\beta$-Sheet Region of T4 Lysozyme, in TFE and SDS. Biochemistry 36 (38):11525-11533.

Scott DJ, Layfield S, Yan Y, Sudo S, Hsueh AJ, Tregear GW, Bathgate RA (2006) Characterization of novel splice variants of LGR7 and LGR8 reveals that receptor signaling is mediated by their unique low density lipoprotein class A modules. The Journal of biological chemistry 281 (46):34942-34954.

Shabanpoor F, Bathgate RAD, Wade JD, Hossain MA (2013a) C-Terminus of the B-Chain of relaxin-3 is important for receptor activity. PloS one 8 (12):e82567.

Shabanpoor F, Hossain MA, Lin F, Wade JD (2013b) Sequential formation of regioselective disulfide bonds in synthetic peptides with multiple disulfide bonds. Methods in molecular biology 1047:81-87.

Wang X-Y, Guo Y-Q, Shao X-X, Liu Y-L, Xu Z-G, Guo Z-Y (2014) Identification of important residues of insulin-like peptide 5 and its receptor RXFP4 for ligand-receptor interactions. Archives of biochemistry and biophysics 558 (0):127-132. 
Table 1: Characterization of hINSL5 variants by MALDI-TOF mass spectrometry and amino acid analysis.

\begin{tabular}{|l|l|l|l|l|}
\hline $\begin{array}{l}\text { Sr. } \\
\text { No. }\end{array}$ & Peptide & $\begin{array}{l}\text { Observed mass } \\
\text { (Theoretical mass) }\end{array}$ & Overall Yield & $\begin{array}{l}\text { Peptide } \\
\text { Content }\end{array}$ \\
\hline 1 & hINSL5-acid & $5047.18(5044.78)$ & $0.8 \%$ & $26.61 \%$ \\
\hline 3 & hINSL5-B-acid & $5046.12(5043.80)$ & $3.2 \%$ & $75.39 \%$ \\
\hline 4 & hINSL5-amide & $5043.29(5042.81)$ & $8 \%$ & $45.71 \%$ \\
\hline
\end{tabular}


Table 2: Pooled binding affinity (pKi), cAMP activity $\left(\mathrm{pEC}_{50}\right)$ and ERK1/2 data for hINSL5 variants at RXFP4.

\begin{tabular}{|l|l|l|l|l|}
\hline INSL5 variants & Binding (pKi) & $\mathbf{N}$ & cAMP activity $\left(\mathbf{p E C}_{\mathbf{5 0}}\right)$ & $\mathbf{N}$ \\
\hline hINSL5-acid & $7.38 \pm 0.04$ & 4 & $8.34 \pm 0.22$ & 3 \\
\hline hINSL5-B-acid & $7.31 \pm 0.08$ & 5 & $7.78 \pm 0.01^{*}$ & 3 \\
\hline hINSL5-amide & $<6$ & & & 3 \\
& & 4 & $<5$ & \\
\hline
\end{tabular}

* p <0.05 vs hInsl5-acid 


\section{Figure Legends}

Figure 1. Primary structure of human INSL5 analogues: hINSL5-acid, hINSL5-B-acid and hINSL5 amide. C-terminal modifications are highlighted

Figure 2. Comparison of in vitro RXFP4 receptor bioassays of hINSL5-B-acid and hINSL5-amide with hINSL5-acid: (A) competition binding curve of analogues competing with $5 \mathrm{nM}$ of Eu-(A)-mINSL5; (B) cAMP inhibition assay of hINSL5-B-acid, hINSL5-amide and hINSL5-acid. The data are the result of $n=3-4$ independent experiments and are expressed as mean \pm SEM.

Figure 3. Comparison of in vitro RXFP4 receptor p-ERK1/2 activity of hINSL5-B-acid and hINSL5-amide with hINSL5-acid: Concentration-dependent activation of ERK1/2 phosphorylation: The data are the result of $\mathrm{n}=3-4$ independent experiments and are expressed as mean \pm SEM. 
Figure 1

$$
\begin{aligned}
& \mathbf{Z}-\mathbf{D}-\mathbf{L}-\mathbf{Q}-\mathbf{T}-\mathbf{L}-\overline{C-C}-\mathbf{T}-\mathbf{D}-\mathbf{G}-\mathbf{C}-\mathbf{S}-\mathbf{M}-\mathbf{T}-\mathbf{D}-\mathbf{L}-\mathbf{S}-\mathbf{A}-\mathbf{L}-\mathbf{C}-\mathrm{OH} \\
& \mathbf{H}-\mathbf{K}-\mathbf{E}-\mathbf{S}-\mathbf{V}-\mathbf{R}-\mathbf{L}-\mathbf{C}-\mathbf{G}-\mathbf{L}-\mathbf{E}-\mathbf{Y}-\mathbf{I}-\mathbf{R}-\mathbf{T}-\mathbf{V}-\mathbf{I}-\mathbf{Y}-\mathbf{I}-\mathbf{C}-\mathbf{A}-\mathbf{S}-\mathbf{S}-\mathbf{R}-\mathbf{W O H}
\end{aligned}
$$

hINSL5-acid

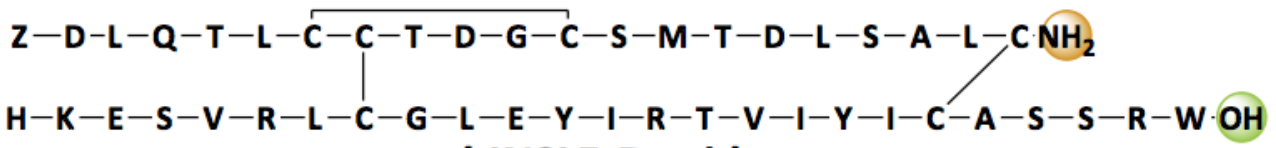
hINSL5-B-acid

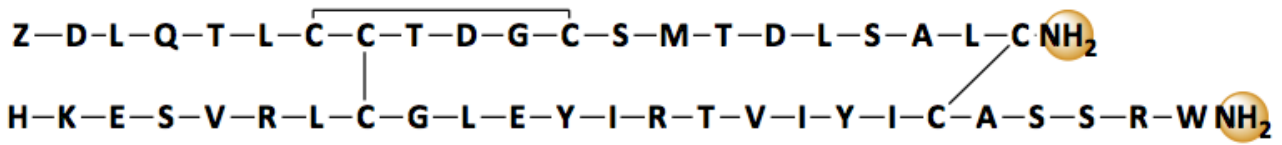
hINSL5-amide 
Figure 2

a) Eu-INSL 5 competition binding

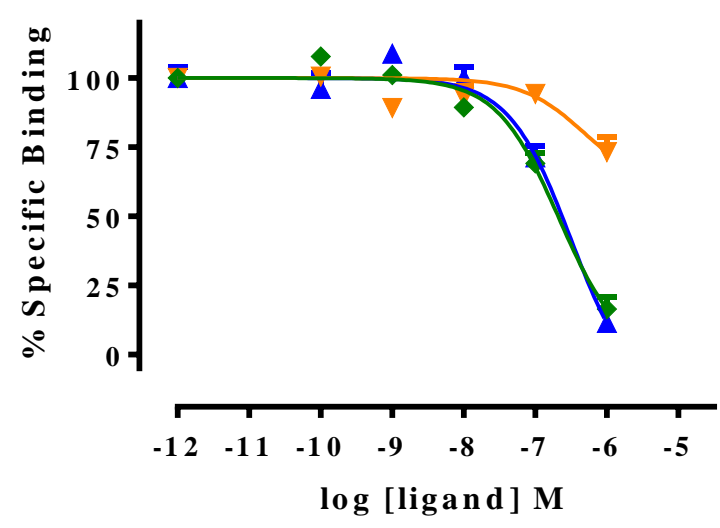

\ INSL5-acid

$\leadsto$ INSL5-B-acid

$\rightarrow$ INSL5-amide

b) RX F P 4 cA M P activity

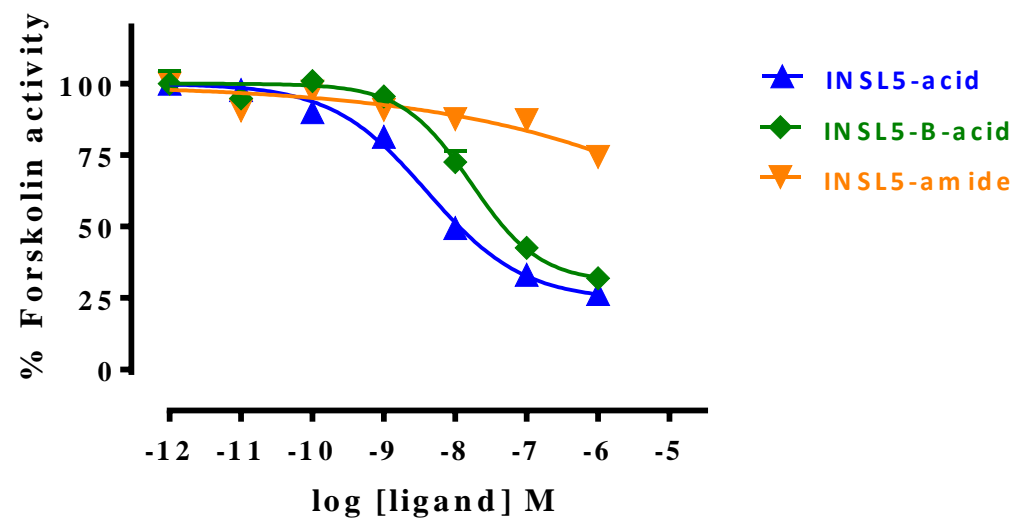


Figure 3

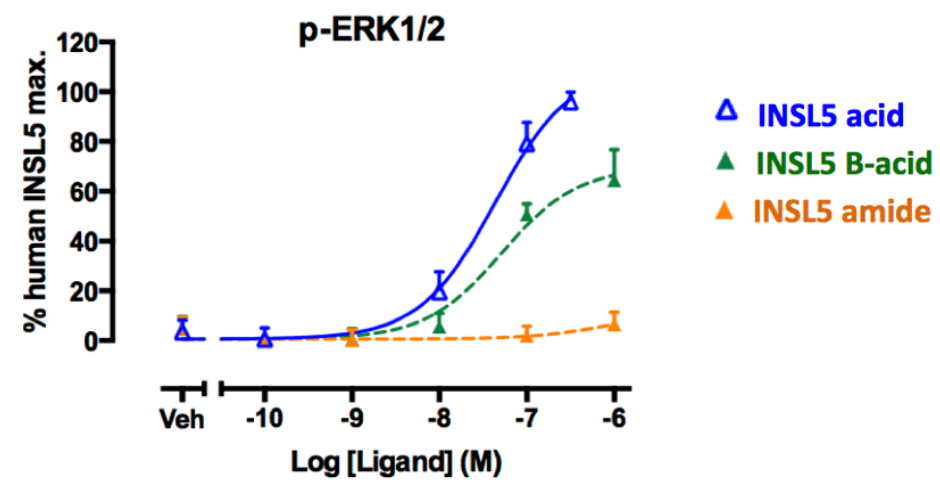

